\title{
ON THE TIGHTNESS OF THE ZHANG-YEUNG INEQUALITY FOR GAUSSIAN VECTORS*
}

\author{
RADIM LNĚNIČKA ${ }^{\dagger}$
}

\begin{abstract}
The Zhang-Yeung unconditional inequality for Shannon entropies of discrete random variables can be extended to Gaussian variables. A necessary and sufficient condition for equality in the resulting inequality is presented.
\end{abstract}

Mathematics subject classification. Primary: 94A17. Secondary: 15A15

Keywords. Entropy; Inequality; Information measure; Mutual information

1. Introduction. Z. Zhang and R. W. Yeung [5] found the following remarkable inequality

$$
\begin{aligned}
I(Z ; U)-I(Z ; U \mid X)-I(Z ; U \mid Y) \leq & \frac{1}{2}[I(X ; Y)+I(X ; Z, U) \\
& +I(Z ; U \mid X)-I(Z ; U \mid Y)],
\end{aligned}
$$

valid for four random variables $X, Y, Z, U$ taking finite number of values. Here $I$ denotes the mutual information. By continuity, the inequality holds also for more general vectors, especially for Gaussian ones. Generally, it is very difficult to identify the cases when the equality takes place, in this note we give the solution of this problem for Gaussian vectors. Please refer to [4] for a comprehensive treatment of the subject.

2. Preliminaries. Let $N=\{1,2, \ldots, n\}$ and $I, J \subseteq N$. For any matrix $A=$ $\left(a_{i, j}\right)_{i, j \in N}$, its submatrix $\left(a_{i, j}\right)_{i \in I, j \in J}$ is denoted by $A_{I, J}$ and $A_{I}=A_{I, I}$. The term $I J$ is used as an abbreviation of $I \cup J$. The relation $I \prec J$ means that the maximal element of $I$ is less than the minimal element of $J$. The determinant of $A$ is denoted by $|A|$ and, by convention, $\left|A_{\emptyset}\right|=1$. Once $I, J$ are disjoint and nonempty and $A_{J}$ is invertible, $\left(A_{I J} \mid A_{J}\right)=A_{I}-A_{I, J}\left(A_{J}\right)^{-1} A_{J, I}$ defines the (generalized) Schur complement of $A_{J}$ in $A_{I J}$. If $J=\emptyset$, define $\left(A_{I J} \mid A_{J}\right)=A_{I}$. The unit matrix is denoted by $E . E_{I}$ is the unit matrix of order $\operatorname{card}(I)$. For a real-valued function $f$ defined on the power set $2^{N}, \square$ and $\triangle$ are defined by the following expressions:

$$
\begin{aligned}
\square f(I, J \mid K, L)= & f(I K)+f(J K)+f(I L)+f(J L)+f(K L)- \\
& -f(I J)-f(K)-f(L)-f(I K L)-f(J K L), \\
\triangle f(I, J \mid K)= & f(I K)+f(J K)-f(I J K)-f(K), I, J, K, L \subseteq N .
\end{aligned}
$$

*Received on January 29, 2003; accepted for publication on May 12, 2003. This work was partially supported by the grant No. A 1075104 of GAAVČR.

${ }^{\dagger}$ Charles University, Faculty of Mathematics and Physics, Prague, Czech Republic. E-mail: lnenicka@csmat.karlin.mff.cuni.cz 
The following lemma was found by F. Matúš [2].

Lemma 1. For $\square$ and $\triangle$, any real-valued function $f$ on $2^{N}$ and $I, J, K, L, \hat{I} \subseteq N$, the following equality is true:

$$
\begin{aligned}
& \square f(I, J \mid K, L)+\triangle f(\hat{I}, K \mid L)+\triangle f(\hat{I}, L \mid K)+\triangle f(K, L \mid \hat{I}) \\
& +[\triangle f(I, \hat{I} \mid K L)+\triangle f(J, \hat{I} \mid K L)+\triangle f(I J, \hat{I} \mid K L)] \\
= & \triangle f(I, \hat{I} \mid K)+\triangle f(J, \hat{I} \mid K)+\triangle f(I, \hat{I} \mid L)+\triangle f(J, \hat{I} \mid L)+\triangle f(I, J \mid \hat{I}) \\
& +\triangle f(K, L \mid I \hat{I})+\triangle f(K, L \mid J \hat{I})+\triangle f(K L, \hat{I} \mid I J) .
\end{aligned}
$$

A straightforward proof is omitted.

Lemma 2. Let $A=A_{N}$ be a positive definite matrix. Then, for any disjoint subsets $I, J, K \subseteq N$,

$$
\left|A_{I K}\right|\left|A_{J K}\right| \geq\left|A_{I J K}\right|\left|A_{K}\right|
$$

If $I$ and $J$ are nonempty, the equality is attained if and only if $\left(A_{I J K} \mid A_{K}\right)_{I, J}=0$.

Proof. Let both $I$ and $J$ be nonempty sets. Without loss of generality, suppose $I \prec J \prec K$. Then everything follows from Hadamard's inequality applied to the matrix $\left(A_{I J K} \mid A_{K}\right)$ and from the relations $\left|A_{I K}\right|=\left|A_{K}\right|\left|\left(A_{I K} \mid A_{K}\right)\right|,\left|A_{J K}\right|=$ $\left|A_{K}\right|\left|\left(A_{J K} \mid A_{K}\right)\right|,\left|A_{I J K}\right|=\left|A_{K}\right|\left|\left(A_{I J K} \mid A_{K}\right)\right|$. For Hadamard's inequality see [3], p. 146.

3. The Zhang-Yeung Inequality for Gaussian Vectors. The Zhang-Yeung inequality (1) can be equivalently rewritten as

$$
\square h(1,2 \mid 3,4)+\triangle h(1,3 \mid 4)+\triangle h(1,4 \mid 3)+\triangle h(3,4 \mid 1) \geq 0
$$

where $h$ denotes the entropy function of a discrete random vector $\left(X_{1}, X_{2}, X_{3}, X_{4}\right)$ and braces are omitted.

Keeping in mind the fact that the entropy of an $n$-dimensional Gaussian vector with positive definite covariance matrix $A$ is given by $\frac{1}{2} \log \left[(2 \pi e)^{n}|A|\right]$ (see [1]), this inequality can be reformulated for Gaussian vectors and also a necessary and sufficient condition for attaining of the equality in this case can be given.

THEOREM 1. For every nonnegative definite matrix $A=A_{N}$ with $N$ partitioned into disjoint sets $I, J, K$ and $L$,

$$
\left|A_{I}\right|\left|A_{K}\right|^{2}\left|A_{L}\right|^{2}\left|A_{I J}\right|\left|A_{I K L}\right|^{4}\left|A_{J K L}\right| \leq\left|A_{I K}\right|^{3}\left|A_{I L}\right|^{3}\left|A_{J K}\right|\left|A_{J L}\right|\left|A_{K L}\right|^{3} .
$$

If $A$ is positive definite and $h_{A}(S)=\log \left|A_{S}\right|$, for any $S \subseteq N$, inequality (2) is equivalent with

$$
\square h_{A}(I, J \mid K, L)+\triangle h_{A}(I, K \mid L)+\triangle h_{A}(I, L \mid K)+\triangle h_{A}(K, L \mid I) \geq 0 .
$$

Here, the equality takes place if and only if

$$
A_{I, J K L}=0, A_{K, L}=0 \text { and }\left(A_{K L J} \mid A_{J}\right)_{K, L}=0 .
$$


REMARK 1. If $A$ is the covariance matrix of a Gaussian random vector $X_{N}$, with multivariate components $X_{I}, X_{J}, X_{K}$ and $X_{L}$, condition (4) is equivalent to the conditional independences $X_{I} \perp\left(X_{J}, X_{K}, X_{L}\right), X_{K} \perp X_{L}$ and $X_{K} \perp X_{L} \mid X_{J}$.

Proof of Theorem 1. By continuity, one can restrict in (2) to positive definite matrices. The equivalence of (2) and (3) is straightforward. Without any loss of generality, suppose $K \prec L \prec I \prec J$. Having a positive definite matrix $A$, let $D_{A}$ be the block diagonal matrix with the blocks $\left(A_{K}\right)^{-\frac{1}{2}},\left(A_{L}\right)^{-\frac{1}{2}},\left(A_{I}\right)^{-\frac{1}{2}},\left(A_{J}\right)^{-\frac{1}{2}}$. It is not difficult to see that the matrix $B=D_{A} A D_{A}$ is positive definite, and $E_{K}, E_{L}, E_{I}, E_{J}$ are the diagonal blocks of $B$. Further, $\left|A_{I J}\right|=\left|B_{I J}\right|\left|A_{I}\right|\left|A_{J}\right|$ and analogously for all unions of subsets of $\{I, J, K, L\}$. Therefore, inequality (3) holds for $A$ if and only if it holds for $B$. Moreover, condition (4) holds for $A$ if and only if it holds for $B$, due to Lemma 2 .

From now on, suppose that $A$ has the form

$$
A=\left(\begin{array}{cccc}
E_{K} & d & c & b \\
d^{T} & E_{L} & f & e \\
c^{T} & f^{T} & E_{I} & a \\
b^{T} & e^{T} & a^{T} & E_{J}
\end{array}\right)
$$

Define

$$
C=\left(\begin{array}{ccc}
A & & A_{K L, I} \\
A_{I, K L} & P^{T} & A_{I}
\end{array}\right)=\left(\begin{array}{ccccc} 
& & & & \\
& & & \\
& & & \\
& & & \\
& & & \\
& & & \\
c^{T} & f^{T} & x^{T} & y^{T} & E_{I}
\end{array}\right),
$$

where

(5)

$$
P=A_{I J, K L}\left(A_{K L}\right)^{-1} A_{K L, I}=\left(\begin{array}{c}
x \\
y
\end{array}\right)=\left(\begin{array}{cc}
c^{T} & f^{T} \\
b^{T} & e^{T}
\end{array}\right)\left(\begin{array}{cc}
E_{K} & d \\
d^{T} & E_{L}
\end{array}\right)^{-1}\left(\begin{array}{c}
c \\
f
\end{array}\right) .
$$

The set of bottom $\operatorname{card}(I)$ rows of $C$ will be indexed by $\hat{I}$. Note that

$$
C_{K L I J}=A, C_{\hat{I}}=A_{I} \text { and } C_{K L \hat{I}}=A_{K L I} .
$$

It can be easily checked that $C=U D U^{T}$, where

$$
U=\left(\begin{array}{ccc}
E_{K L} & 0 & 0 \\
A_{I J, K L}\left(A_{K L}\right)^{-1} & E_{I J} & 0 \\
A_{I, K L}\left(A_{K L}\right)^{-1} & 0 & E_{I}
\end{array}\right)
$$

and

$$
D=\left(\begin{array}{ccc}
A_{K L} & 0 & 0 \\
0 & \left(A \mid A_{K L}\right) & 0 \\
0 & 0 & \left(A_{I K L} \mid A_{K L}\right)
\end{array}\right)
$$


Since the diagonal blocks of $D$ are positive definite (see [3], p. 155) and $U$ is an invertible matrix, the matrix $C$ is positive definite. By the construction of $C$,

$$
\left(C \mid C_{K L}\right)_{I J, \hat{I}}=\left(\begin{array}{c}
\left(C_{I \hat{I} K L} \mid C_{K L}\right)_{I, \hat{I}} \\
\left(C_{J \hat{I} K L} \mid C_{K L}\right)_{J, \hat{I}}
\end{array}\right)=P-A_{I J, K L}\left(A_{K L}\right)^{-1} A_{K L, I}=0
$$

what implies

$$
\triangle h_{C}(I J, \hat{I} \mid K L)=\triangle h_{C}(I, \hat{I} \mid K L)=\triangle h_{C}(J, \hat{I} \mid K L)=0
$$

by Lemma 2 . When Lemma 1 is applied to the function $h_{C}$, the eight terms of the right-hand side of the resulting identity are nonnegative, the bracket vanishes by (7) and thus

$$
\square h_{C}(I, J \mid K, L)+\triangle h_{C}(\hat{I}, K \mid L)+\triangle h_{C}(\hat{I}, L \mid K)+\triangle h_{C}(K, L \mid \hat{I}) \geq 0 .
$$

Inequality (3) now follows from (6).

The equality in (3) holds if and only if the eight terms vanish. Using Lemma 2 this takes place if and only if the blocks of the matrix $C$ satisfy the following system of matrix equations:

$$
\begin{aligned}
& x=c^{T} c \\
& \left(\text { from } \triangle h_{C}(I, \hat{I} \mid K)=0\right) \\
& y=b^{T} c \\
& x=f^{T} f \\
& y=e^{T} f \\
& \left(\text { from } \triangle h_{C}(J, \hat{I} \mid K)=0\right) \\
& a=x y^{T} \\
& d=\left(\begin{array}{cc}
c & c
\end{array}\right)\left(\begin{array}{cc}
E_{I} & x \\
x^{T} & E_{I}
\end{array}\right)^{-1}\left(\begin{array}{c}
f^{T} \\
f^{T}
\end{array}\right) \quad\left(\text { from } \triangle h_{C}(K, L \mid I \hat{I})=0\right) \\
& d=\left(\begin{array}{ll}
b & c
\end{array}\right)\left(\begin{array}{cc}
E_{J} & y \\
y^{T} & E_{I}
\end{array}\right)^{-1}\left(\begin{array}{c}
e^{T} \\
f^{T}
\end{array}\right) \quad\left(\text { from } \triangle h_{C}(K, L \mid J \hat{I})=0\right) \\
& \left(\begin{array}{c}
c \\
f
\end{array}\right)=\left(\begin{array}{cc}
c & b \\
f & e
\end{array}\right)\left(\begin{array}{cc}
E_{I} & a \\
a^{T} & E_{J}
\end{array}\right)^{-1}\left(\begin{array}{l}
x \\
y
\end{array}\right) \quad\left(\text { from } \triangle h_{C}(K L, \hat{I} \mid I J)=0\right) \text {. }
\end{aligned}
$$

If $A$ satisfies (4), i.e. $a=0, c=0, d=0, f=0$ and $b e^{T}=0$, then $x=0$ and $y=0$ by (5) and (8) holds. Hence (4) is sufficient for the equality in (3).

For the necessity, let the blocks of $A$ (and $x, y$ in $C$ constructed from $A$ ) satisfy the system of equations (8). By [3], p. 17,

$$
\left(\begin{array}{cc}
E_{K} & d \\
d^{T} & E_{L}
\end{array}\right)^{-1}=\left(\begin{array}{cc}
E_{K} & 0 \\
0 & 0
\end{array}\right)+\left(\begin{array}{c}
d \\
-E_{L}
\end{array}\right) s^{-1}\left(\begin{array}{ll}
d^{T} & -E_{L}
\end{array}\right),
$$


where $s=E_{L}-d^{T} d$ is the Schur complement of $E_{K}$ in $\left(\begin{array}{cc}E_{K} & d \\ d^{T} & E_{L}\end{array}\right)$. By (5),

$$
x=\left(\begin{array}{cc}
c^{T} & f^{T}
\end{array}\right)\left(\begin{array}{cc}
E_{K} & d \\
d^{T} & E_{L}
\end{array}\right)^{-1}\left(\begin{array}{c}
c \\
f
\end{array}\right) .
$$

Using (9) and $x=c^{T} c$,

$$
x=x+\left(d^{T} c-f\right)^{T} s^{-1}\left(d^{T} c-f\right) .
$$

Since the matrix $s$ is positive definite, $d^{T} c=f$. Multiplying the sixth equation of (8) by $c^{T}$ from the left and by $f$ from the right,

$$
x=\left(\begin{array}{ll}
x & x
\end{array}\right)\left(\begin{array}{cc}
E_{I} & x \\
x^{T} & E_{I}
\end{array}\right)^{-1}\left(\begin{array}{l}
x \\
x
\end{array}\right) .
$$

Since $x=c^{T} c, x$ is symmetric. Similarly as in (9),

$$
\left(\begin{array}{cc}
E_{I} & x \\
x & E_{I}
\end{array}\right)^{-1}=\left(\begin{array}{cc}
E_{I} & 0 \\
0 & 0
\end{array}\right)+\left(\begin{array}{c}
x \\
-E_{I}
\end{array}\right) t^{-1}\left(\begin{array}{ll}
x & -E_{I}
\end{array}\right)
$$

where $t=E_{I}-x^{2}$. Combining (10) and (11),

$$
x=x^{2}+\left(x^{2}-x\right) t^{-1}\left(x^{2}-x\right) .
$$

This implies

$$
0=\left(x^{2}-x\right) t^{-1}\left(t+x^{2}-x\right)=-x\left(E_{I}-x\right) t^{-1}\left(E_{I}-x\right) .
$$

As $t=\left(E_{I}+x\right)\left(E_{I}-x\right)$ is invertible, the matrix $E_{I}-x$ is regular. Multiplying equality (12) by $\left(E_{I}-x\right)^{-1} t\left(E_{I}-x\right)^{-1}$ from right, we arrive at $x=0$. Now, from (8) it is easy to see that all $a, c, d, f$ are the zero blocks, thus

$$
A=\left(\begin{array}{cccc}
E_{K} & 0 & 0 & b \\
0 & E_{L} & 0 & e \\
0 & 0 & E_{I} & 0 \\
b^{T} & e^{T} & 0 & E_{J}
\end{array}\right) .
$$

Since also $y=0$, the seventh equation of (8) gives $b e^{T}=0$. Therefore (4) holds for $A$.

REMARK 2. If the equality holds in (3), all four summands of its left-hand side are zero due to (4).

Acknowledgement. I am very much indebted to my supervisor František Matúš for introducing me to the subject, providing me the unpublished manuscript and for many valuable suggestions for improvements. 
[1] T. M. Cover, J. A. Thomas, Determinant Inequalities via Information Theory, SIAM Journal on Matrix Analysis and Applications, 9(1988), pp. 384-392.

[2] F. Matúš, On Selfadhesive Polymatroids, Unpublished manuscript.

[3] V. V. Prasolov, Problems and Theorems in Linear Algebra, American Mathematical Society, USA, 1996.

[4] R. W. Yeung, A First Course in Information Theory. Kluwer Academic/Plenum Publisher, 2002.

[5] Z. Zhang, R. W. Yeung, On Characterization of Entropy Function via Information Inequalities, IEEE Transactions on Information Theory, 44(1998), pp. 1440-1452. 